\title{
Kinematic Condition for Maximizing the Thrust of a Robotic Fish Using a Compliant Caudal Fin
}

\author{
Yong-Jai Park, Student Member, IEEE, Useok Jeong, Student Member, IEEE, Jeongsu Lee, \\ Seok-Ryung Kwon, Ho-Young Kim, and Kyu-Jin Cho, Member, IEEE
}

\begin{abstract}
The compliance of a fin affects the thrust of underwater vehicles mimicking the undulatory motion of fish. Determining the optimal compliance of a fin to maximize thrust is an important issue in designing robotic fish using a compliant fin. We present a simple method to identify the condition for maximizing the thrust generated by a compliant fin propulsion system. When a fin oscillates in a sinusoidal manner, it also bends in a sinusoidal manner. We focus on a particular kinematic parameter of this motion: the phase difference between the sinusoidal motion of the driving angle and the fin-bending angle. By observing the relationship between the thrust and phase difference, we conclude that while satisfying the zero velocity condition, the maximum thrust is obtained when a compliance creates a phase difference of approximately $\pi / 2$ at a certain undulation frequency. This half-pi phase delay condition is supported by thrust measurements from different compliant fins (four caudal-shaped fins with different aspect ratios) and a beam bending model of the compliant fin. This condition can be used as a guideline to select the proper compliance of a fin when designing a robotic fish.
\end{abstract}

Index Terms-Compliant fin, flapping, flexible fin, flexible foil, half-pi phase delay, maximum thrust, pseudo-rigid-body model, robotic fish, underwater robot.

\section{INTRODUCTION}

$\mathbf{U}$ NDERWATER vehicles have used various mechanisms in an attempt to mimic the motion of fish and aquatic mammals, such as undulating body motion and oscillating caudal fin motion. Biomimetic propulsion creates a traveling wave, which is known to yield better power efficiency and turning performance than that of screw propulsion [1].

Various mechanisms using multilinks have been developed to mimic the undulating motion of fish. Using six servo motors and eight linkages, Barrett built RoboTuna, which was the first

Manuscript received March 6, 2012; accepted June 15, 2012. Date of publication July 10, 2012; date of current version December 3, 2012. This paper was recommended for publication by Associate Editor A. Ijspeert and Editor W. K. Chung upon evaluation of the reviewers' comments. This work was supported by the Basic Science Research Program and the Converging Research Center Program through the National Research Foundation of Korea, funded by the Ministry of Education, Science and Technology under Grant 2009-0087640, Grant 2009-0070058, and Grant 2009-0082824.

Y.-J. Park, U. Jeong, S.-R. Kwon, and K.-J. Cho are with the BioRobotics Laboratory, School of Mechanical and Aerospace Engineering/IAMD, Seoul National University, Seoul 151-742, Korea (e-mail: yjlone76@snu.ac.kr; snopyy@snu.ac.kr; kwons777@snu.ac.kr; kjcho@snu.ac.kr).

J. Lee and H.-Y. Kim are with the Micro Fluid Mechanics Laboratory, School of Mechanical and Aerospace Engineering/IAMD, Seoul National University, Seoul 151-742, Korea (e-mail: sabaham1@snu.ac.kr; hyk@snu.ac.kr).

Color versions of one or more of the figures in this paper are available online at http://ieeexplore.ieee.org.

Digital Object Identifier 10.1109/TRO.2012.2205490 biomimetic robotic fish [2]. Morikawa et al. built a robotic fish that mimics the caudal muscle-skeletal structure of tuna, using two air rubber artificial muscles and a multijoint bending mechanism [3]. A robotic dolphin was designed with four links and six servo motors to mimic the dorsoventral movement of a real dolphin [4]. Low developed a fish robot to generate arbitrary undulating waveforms, by connecting ten servo motors in series by linking them with sliders [5].

Conversely, several researchers have experimented with using compliant material in their mechanisms. Compliance simply creates undulating motion without linkages. Lauder et al. [6], [7] investigated the propulsion of fish locomotion under various motions of the pectoral and caudal fins. They showed that a cupping motion is more effective than other forms of locomotion, e.g., flat motion, W-shaped motion, rolling motion, or undulation. In addition, Lauder et al. used a flexible plastic foil to explore the effects of changing swimming speed, foil length, and shape of the foil-trailing edge on undulatory locomotion [8]. Alvarado et al. designed a robotic fish with a simple and robust mechanism, using a compliant body that was approximated by a continuous cantilever beam to generate a fish-like oscillating motion [9], [10]. This simple design achieved biomimetic locomotion using only one servo motor, while most other robotic fish use several motors to achieve biomimetic modes of swimming. Daou et al. used Alvarado's compliant body model to derive the relationship between an actuation moment and the resulting lateral deflection of a fish tail [11]. Several fish robots that use smart actuators to create undulating motion have also been investigated [12].

Moreover, an experimental study was conducted on the effect of compliance on the thrust of a propulsion system [13]. To test the effect of compliance, a rigid fin with a spring was used as the compliant tail of a propulsion system. Thrust was measured while varying the frequency, amplitude, aspect ratio (AR), spring constant, and water flow velocity. Because fin elasticity affects the maximum thrust, an elastic fin can improve efficiency by more than $27 \%$, compared with a rigid fin [14]. Lauder and Madden reported that compliance is important for vectoring forces and generating thrust [15]. They explain that flexibility results in the generation of a chordwise wave, which for sunfish may produce thrust during the transition from abduction to adduction [16]. Vanella et al. reported that the flexibility of wings of flying creatures enables a passive pitching motion, which induces a phase difference between a leading edge and a trailing edge [17].

During swimming, the cambering of flukes of a cetacean can increase the production of hydrodynamic forces, particularly 
during direction reversal in the oscillatory cycle [18]. Ahlborn et al. studied the effect of tail stiffness in the fast-start swimming of fish [19], [20]. It was discovered that a slightly flexible tail generates the highest impulse related to thrust generation. Kim and Gharib used fins with different shapes and stiffness to study the relationship between vortex formation and thrust performance [21]. To increase the thrust of the robotic fish, changing the stiffness of the fin should be considered. Kobayashi et al. attempted to develop a variable-stiffness fin with a variableeffective-length spring [22]. Huh et al. designed a stiffness adjustable structure that changes its stiffness by pulling a tendon [23]. This stiffness adjustable structure was designed to be used for a compliant fin to increase the thrust of the robotic fish. Although there is evidence that changing the compliance results in a change in the thrust generated by the propulsion system, there is no guideline on how to choose compliance to maximize thrust.

Various models of thrust generated by fish have been presented. Wu proposed a modified thin airfoil theory to analyze the motion of a waving plate in a 2-D potential flow [24]. Lighthill introduced an elongated-body theory to model the reactive force exerted on a fish when moving in water [25]. Both methods can be used to calculate thrust. For example, the steady-state speed of a robotic fish built using an ionic polymer-metal composite (IPMC) was estimated using the Lighthill model, which took into account the IPMC actuator dynamics [26]. Numerical and statistical methods can be used to compute the thrust of a fish. The thrust of a robotic fish with an undulating fin was computed using numerical methods [27]. Using a dimensional statistical method, the thrust and swimming speed of robotic fish were estimated with various kinematic parameters [28].

However, using these approaches to compute the thrust generated by the undulating fin requires parameters directly related to the fluid flow, e.g., swimming speed and kinematics of the undulating fin. Swimming speed can be determined by modeling the problem as a fluid-structure interaction (FSI) problem, which is a numerical method that requires large amounts of computational resources and time to solve [29]. To obtain the optimal design of a compliant fin analytically, both the swimming speed and kinematics are required for the calculation of the thrust generated by the fin. However, current compliant fin modeling approaches using FSI require knowledge of the kinematics of the compliant fin or body under certain conditions; kinematics is typically derived from experimental measurements [29], [30]. Therefore, obtaining the optimal design of a compliant fin requires a large amount of experiments.

In this paper, we show that while the zero velocity condition is satisfied, a kinematic condition exists that maximizes the thrust of a propulsion system with a compliant fin. When a fin oscillates in a sinusoidal manner, the fin also bends in a sinusoidal manner. We focus on a kinematic parameter of this motion: the phase difference between the sinusoidal motion of the driving angle and the fin-bending angle. The phase difference is zero for rigid fins, and increases as compliance increases. We conduct experiments while varying the following parameters: 1) shape of the caudal fin (four shapes of equal area are used); 2) stiffness of the caudal fin; and 3) oscillating frequency. The results

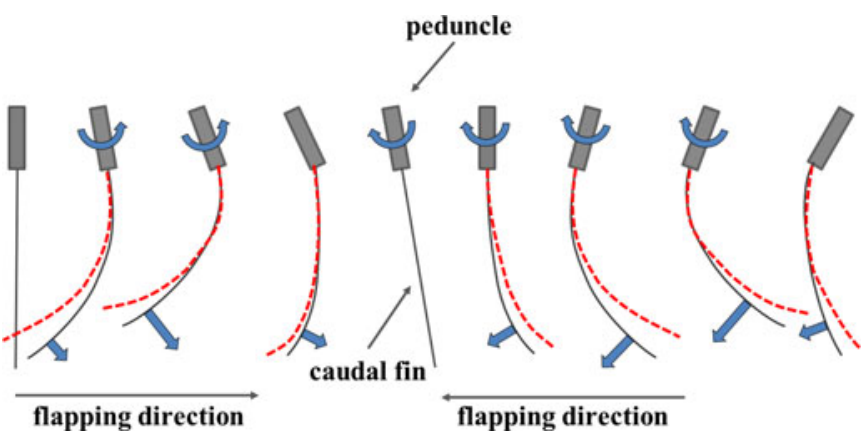

Fig. 1. Schematic drawing depicting the concept of motion for compliant caudal fins, which is based on the biomimetic motion of fish tails. The dotted and gray straight lines indicate changes in motion due to differences in stiffness.

show that for a certain oscillating frequency, and while the zero velocity condition is satisfied, the compliance that maximizes the thrust induces a phase difference of $\pi / 2$, regardless of the shape of the fin. According to this kinematic condition, the bending angle is maximized when the fin is at its maximum speed. A biological study has shown that dolphins swim with a constant phase difference of approximately $\pi / 6$ in terms of position, which is approximately equivalent to $\pi / 2$ when transformed into an angle [31]. There exists additional evidence that supports our conclusion. Specifically, the Strouhal number of a self-propelled flapping wing is minimized when the phase difference between heaving and pitching is $\pi / 2$ [32]. The thrust of the robotic fish is computed using the bending force of the fin and its kinematic model. The large deflection bending of the fin is modeled using a pseudo-rigid-body model. Using this model, we perform kinematic analysis to ascertain the maximum-thrust condition. This condition can be used as a simple design guideline that can drastically reduce the number of experiments required to find the compliant fin design that maximizes the thrust of a robotic fish.

\section{Kinematics OF A Compliant CAUdal Fin}

In this section, we present a simplified kinematic model of a compliant caudal fin, which is a tail fin connected at the end of a peduncle. The concept of the compliant caudal fin is inspired from the oscillating tail of fish and aquatic mammals, such as tuna, bluegill, and dolphin. Our interest lies in carangiform and thunniform swimmers, which mainly use a caudal fin [33].

Fig. 1 shows a schematic drawing of the motion of a compliant caudal fin. When the peduncle (the narrow part of the body to which the fin is attached) oscillates, the fin follows the motion of the peduncle. When the amount of bending of the fin increases, the compliance of the fin also increases. In Fig. 1, this is depicted as a change in motion from the solid line to the dotted line. Solid lines represent the motion of a stiffer fin, while dotted lines represent the motion of a compliant fin.

Using different compliant fins, we investigated the effects of flexible fins on thrust generation, by measuring the thrust generated by the propulsion system. The caudal fin was constructed from a polyvinyl chloride plate, polypropylene plate, and acrylic plate. This composition of materials enabled us to 


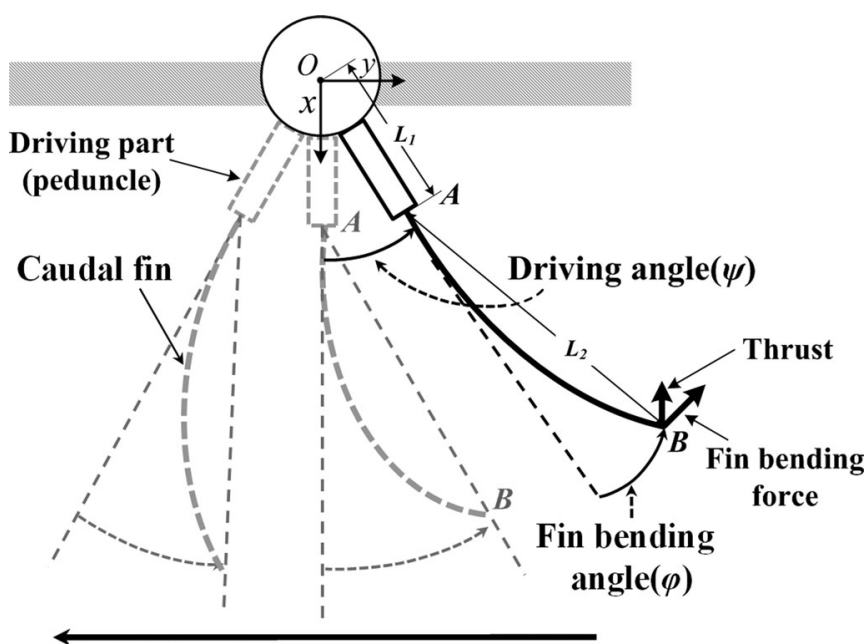

Caudal fin moving direction

Fig. 2. Schematic drawing of the caudal fin connected to the driving part (peduncle). Here, $\varphi$ is the bending angle due to the movement of the caudal fin, and $\psi$ is the driving angle that is driven by the motor.

simplify experimental parameters, and easily replace caudal fins. Moreover, we investigated the effects of the shapes of the fins on thrust generation.

Fig. 2 shows a schematic drawing of the experimental setup used to identify the effects of the compliant caudal fin. Note that the $x$-axis represents the vertical direction of the experimental setup, which would be in the opposite direction of swimming if the caudal fin was attached to the body. The $y$-axis is perpendicular to the body.

The kinematic parameters, driving angle, and fin-bending angle, are defined in the following. Point $O$ is the center of rotation, point $A$ connects the peduncle and the caudal fin, and point $B$ is the endpoint of the caudal fin. The fin-bending angle $\varphi$ is the angle between the straight line $O A$ and line $A B$. We assume that when driven inside water, the caudal fin bends into an arc with a single curvature, and the slope of line $\mathrm{AB}$ is zero at point $A$. Under these assumptions, for a certain fin-bending angle $\varphi$, the endpoint of the caudal fin is unique. The driving angle $\psi$ is the angle between the $x$-axis and line $O A$. Fin-bending angle $\varphi$ and driving angle $\psi$ are positive in the positive $y$-direction.

The caudal fin moves periodically. Thus, because the lateral deflection of a tail at a given point is described by a sinusoidal function [9], we assume that the driving and fin-bending angles also follow a sinusoidal function based on an observation. The driving angle $\psi$ and the fin-bending angle $\varphi$ can be expressed as

$$
\begin{aligned}
& \psi=A_{d} \sin \left(2 \pi f t+\beta_{d}\right) \\
& \varphi=A_{b} \sin \left(2 \pi f t+\beta_{b}\right)
\end{aligned}
$$

where $f$ is the frequency of the oscillating caudal fin, $A_{b}$ is the maximum fin-bending angle, $A_{d}$ is the maximum driving angle, $\beta_{b}$ is the phase of the fin-bending angle, and $\beta_{d}$ is the phase of the driving angle.

In order to evaluate the motion of the caudal fin, we define the phase difference $(\xi)$ as the periodic difference between the
TABLE I

\begin{tabular}{|c|c|c|c|}
\hline $\begin{array}{l}\text { Freq. } \\
(\mathrm{Hz})\end{array}$ & $\begin{array}{c}\text { Materials of Tail and } \\
\text { Thickness }\end{array}$ & $\begin{array}{l}\text { Abbreviation } \\
\text { of Tail }\end{array}$ & $\begin{array}{c}\text { Stiffness } \\
(\mathrm{N} / \mathrm{m})\end{array}$ \\
\hline 0.5 & Polyvinyl chloride $(0.4 \mathrm{~mm})$ & $\mathrm{T} 1$ & 102 \\
\hline 1.0 & Polypropylene $(0.5 . \mathrm{mm})$ & $\mathrm{T} 2$ & 171 \\
\hline 1.6 & Polyvinyl chloride $(0.55 \mathrm{~mm})$ & $\mathrm{T} 3$ & 236 \\
\hline 2.2 & Polypropylene $(0.7 \mathrm{~mm})$ & $\mathrm{T} 4$ & 292 \\
\hline 2.7 & Polyvinyl chloride $(0.75 \mathrm{~mm})$ & $\mathrm{T} 5$ & 775 \\
\hline \multirow[t]{4}{*}{3.2} & Polypropylene $(1.0 \mathrm{~mm})$ & T6 & 803 \\
\hline & Polyvinyl chloride $(1.0 \mathrm{~mm})$ & $\mathrm{T} 7$ & 1666 \\
\hline & Polypropylene $(1.3 \mathrm{~mm})$ & $\mathrm{T} 8$ & 1950 \\
\hline & Acryl $(1.7 \mathrm{~mm})$ & T9 & 8780 \\
\hline
\end{tabular}

CONTROL PARAMETERS

driving angle and fin-bending angle

$$
\xi=\beta_{d}-\beta_{b} .
$$

In this paper, for the sake of simplicity, we omitted the fact that bending characteristics affect the curvature of the caudal fin, and thus, the curvature changes along its length. Instead, we assumed that the fin bends with a single curvature, and that the slope between the peduncle and fin is equal to zero. If the bending characteristics along the length of the caudal fin are considered, then we can obtain more accurate results. However, the definition of the phase difference can be complicated. Therefore, the definition of the phase difference adopted in this paper, using the driving and fin-bending angles, is adequate to generate a simple guideline.

When a compliant fin is driven inside water, distributed forces are applied on the surface of the fin. These forces bend the compliant fin. The projection of the sum of these forces on the $x$-axis is the thrust. Therefore, the thrust $F_{x}$ that is generated by a compliant caudal fin in the negative $x$-direction can be estimated by finding the force required to bend the fin into a specific shape, or a certain fin-bending angle (from $1^{\circ}$ to $50^{\circ}$ ). Because the mass of the caudal fin is small compared with the effect of the added mass of water, the inertial effects are neglected. Consequently, the compliant fin is equivalent to a force sensor.

\section{EXPERIMENTAL PROCEDURE}

The goal of the experiment was to measure the thrust of the propulsion system, and determine its dependence on the measured phase difference. The experiments were carried out using four types of fin shapes, and for each fin shape we tested nine different stiffness values (36 different fins in total). Moreover, each fin was tested under six different frequencies. Table I shows the actual driving frequency and materials used in the experiment. The frequencies used were selected to avoid the natural frequency of the water tank, which was $1.5 \mathrm{~Hz}$. In addition, a sponge was attached to the wall of the water tank to absorb waves, and reduce the water oscillation near the natural frequency.

The stiffness of the materials was measured using a universal test machine. As shown in Table I, acrylic was the stiffest 
(a)
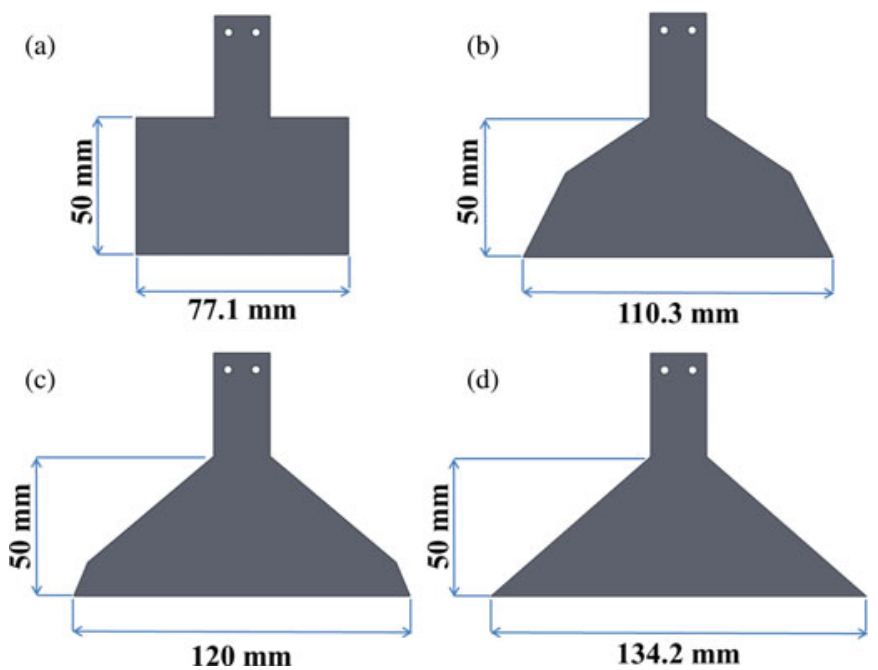

Fig. 3. Shapes of caudal fins. (a) Rectangular fin (F1) with AR 1.5. (b) Delta-shaped fin I (F2) with AR 3.2. (c) Delta-shaped fin II (F3) with AR 3.7. (d) Triangular fin (F4) with AR 4.7.

TABLE II

SPECIFICATIONS OF CAUDAL Fins

\begin{tabular}{ccc}
\hline \hline & Abbreviation of Fin & Aspect ratio \\
\hline Rectangular Fin & F1 & 1.5 \\
Delta-shaped Fin I & F2 & 3.2 \\
Delta-shaped Fin II & F3 & 3.7 \\
Triangular Fin & F4 & 4.7 \\
\hline \hline
\end{tabular}

material, and polyvinyl chloride (thickness of $0.4 \mathrm{~mm}$ ) was the most flexible material.

To evaluate the effects of different bending characteristics, four different shapes of caudal fin (rectangular, delta-shaped I, delta-shaped II, and triangular) with different ARs were considered. In order to verify the effects of the AR, all fins had the same area of $3855 \mathrm{~mm}^{2}$, and the height of each fin was fixed at $50 \mathrm{~mm}$. The shapes of the caudal fins are presented in Fig. 3, and Table II lists their corresponding ARs. The AR was calculated as

$$
\mathrm{AR}=\frac{w^{2}}{S}
$$

where $w$ is the maximum width of the fin (spanwise length), and $S$ is the area of the fin.

The Reynolds number of the compliant caudal fin was selected to match the Reynolds number of adult fish swimming. In this paper, the Reynolds number is defined as $\operatorname{Re}=L^{2} f / v$, where $L$ is the maximum length of the caudal fin, $f$ is the frequency of motion of the caudal fin, and $v$ is the kinematic viscosity of water. The range of the Reynolds number of the compliant caudal fin is approximately $1.2 \times 10^{3}$ to $8.0 \times 10^{3}$. This is in accordance with the typical $R e$ of adult fish swimming, which ranges between $1.0 \times 10^{3}$ and $5.0 \times 10^{6}$ [33].

To create a sinusoidal oscillating motion, the propulsion system was built using a brushed DC motor and scotch-yoke mechanism [34]. The specifications of the propulsion system are listed in Table III.
TABLE III

SPECIFICATIONS OF THE PROPULSION SYSTEM

\begin{tabular}{cl}
\hline \hline Mass & Approx. 900 g \\
Dimension & $310 \mathrm{~mm} \times 60 \mathrm{~mm} \times 160 \mathrm{~mm}$ \\
Actuator & Maxon DC Motor(RE35) with Encoder \\
Driver & Maxon ADS50/5 Speed Controller \\
Power source & 350W Power Supply \\
Materials & Astile, Acryl \\
\hline \hline
\end{tabular}
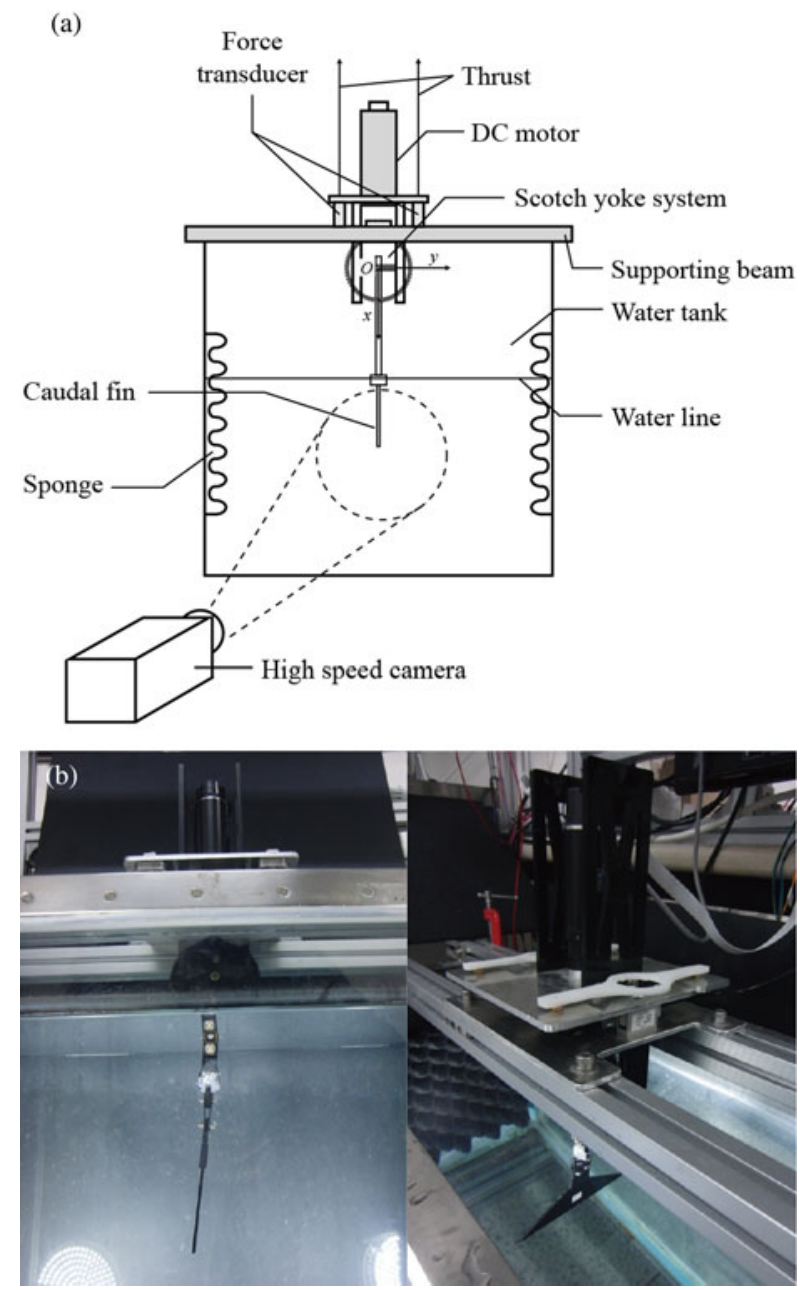

Fig. 4. (a) Layout of experimental setup and driving device with the caudal fin. (b) Actual apparatus.

As shown in Fig. 4, the propulsion system was attached to two force transducers (Ktoyo 333FB) on a water tank. To reduce the vibration of the overall system, the force transducers were installed along the $y$-axis. In order to reduce the effect of flow reflection, the depth of the water tank was five times the length of the fin. The experiments were conducted under the zero velocity condition. However, as shown by Ahlborn et al. [20] and Kim and Gharib [21], these results can be expanded to hold under the fast-start and drag-based conditions. In addition, the aforementioned authors used the experimental results obtained under the zero velocity condition to analyze fast-start swimming and drag-based paddling. 


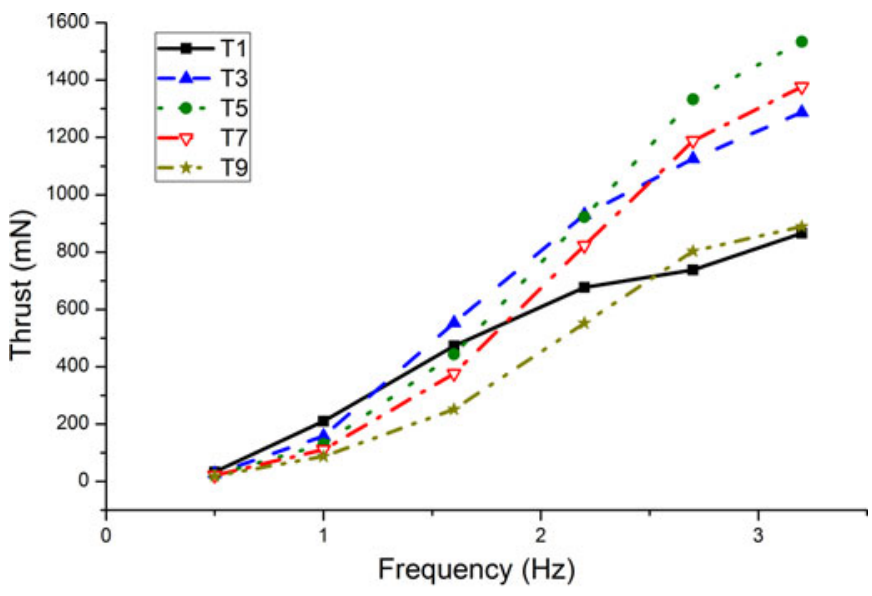

(a)

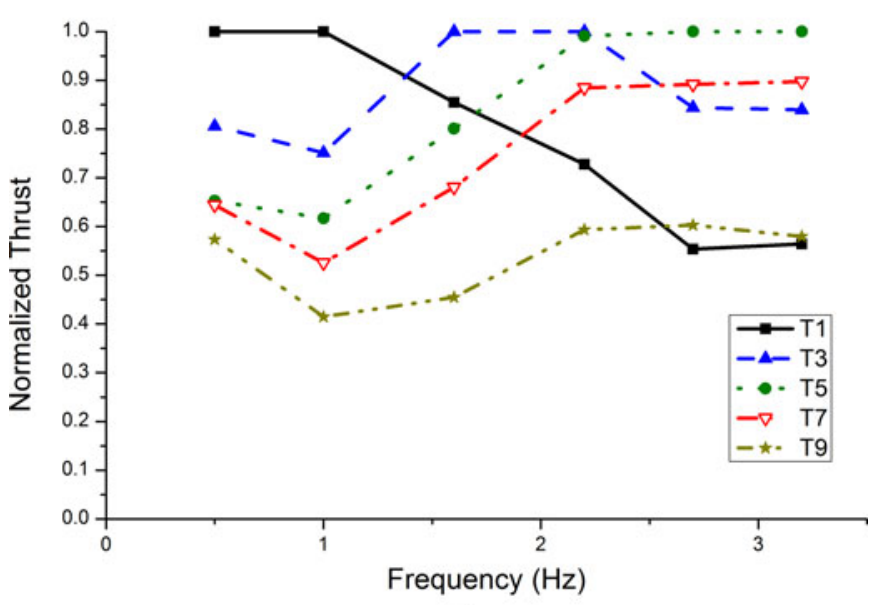

(b)

Fig. 5. (a) Thrust versus frequency for different compliant fins of shape F3 (delta-shaped fin II). (b) Normalized thrust versus frequency for compliant fins of shape F3. As frequency increased, the compliance of the fin that generated the maximum thrust decreased. The measured thrust presented was averaged over one period of oscillation.

Using LabVIEW software installed on a National Instrument CompactRIO DAQ 9172, we measured and processed the force transducer data, which were recorded at 2000 samples/s. Because the two force transducers lay in the $y$-axis, the total force was obtained by summing the two sets of force data. The bending movement of the caudal fin was recorded with a Photron FASTCAM-APX RS high-speed camera at 1000 frames/s.

\section{RESULTS AND ANALYSIS}

\section{A. Thrust Analysis}

The measured thrust was averaged over one period of oscillation. Fig. 5(a) shows the thrust for different compliant fins in relation to frequency, for the case of the delta-shaped fin II (F3). As the frequency increased, the thrust increased for all compliant fins. According to [10], if we had increased the frequency, we would have observed a drop in the thrust. However, because vibration at high frequencies hindered accurate measurements, we limited the driving frequency to below $3.2 \mathrm{~Hz}$. It is emphasized that the focus of this paper is to identify the compliance that
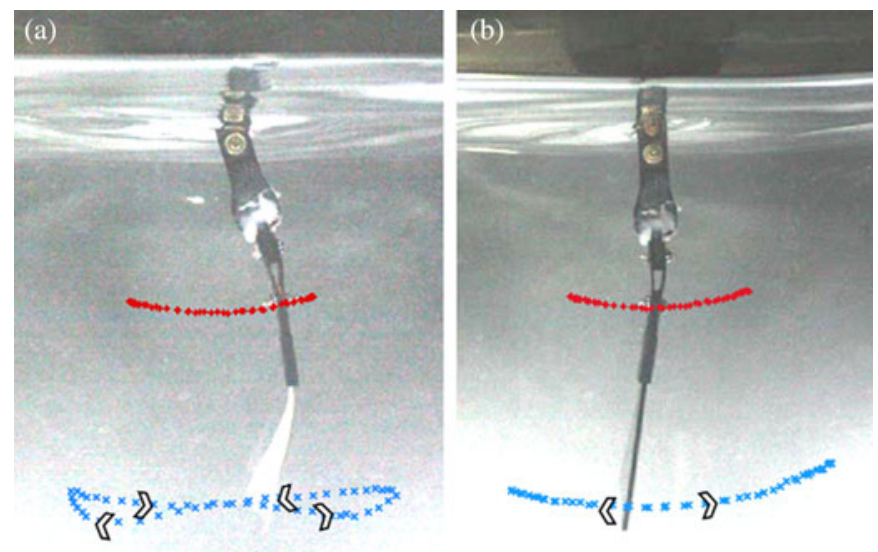

Fig. 6. Results of the delta-shaped fin II (F3) obtained while tracking over one cycle at $2.2 \mathrm{~Hz}$. (a) Maximum thrust (T3). (b) Minimum thrust (T9). In the case of maximum thrust, the figure-eight motion is observed, while it is absent in the case of minimum thrust.

generates the maximum thrust for different oscillating frequencies of the fin, under the zero velocity and fast-start conditions. To better show which compliant fin generated the maximum thrust at each frequency, the thrust values averaged over one period of oscillation were normalized by dividing them by the maximum thrust measured at each frequency. Using the normalized data shown in Fig. 5(b), we can easily identify which compliant fin generated the maximum thrust at each frequency.

At low frequencies, 0.5 and $1.0 \mathrm{~Hz}$, the fin that generated the maximum thrust was T1. At $2.7 \mathrm{~Hz}$, the fin that generated the maximum thrust was T5. Hence, when the driving frequency of the caudal fin is given, the compliance of the fin that generates the maximum thrust can be determined.

\section{B. Kinematic Analysis}

To understand when the average thrust is maximized, we performed kinematic analysis by capturing and analyzing images of the flapping fin. Image analysis software (Xcites's ProAnalyst) was used to extract the bending and driving angles from the video. We used the tracking system in ProAnalyst to track these angles frame by frame. Then, we compared the thrust data we measured against the results obtained by the recorded images.

The effects of compliance are displayed in Fig. 6. The tip of the caudal fin moved in a figure-eight motion, similar to hovering wings [35]. This hysteresis of the motion is related to the phase difference. When the phase difference increases, the figure-eight motion becomes more prominent. This motion has also been observed in swimming fish [36]. Conversely, a rigid fin yields a zero phase difference, and, hence, creates a typical pendulumlike motion. The arrows in Fig. 6 indicate the direction of the movement of the caudal fin. The fin that generated the figureeight motion yielded larger thrust compared with the fin that did not generate a figure-eight motion. However, the size of the figure-eight motion was not proportional to the thrust yielded.

To identify the kinematic condition that generates the maximum thrust, we compared, for one cycle of oscillation, the driving and fin-bending angles, and plotted the resulting thrust. 


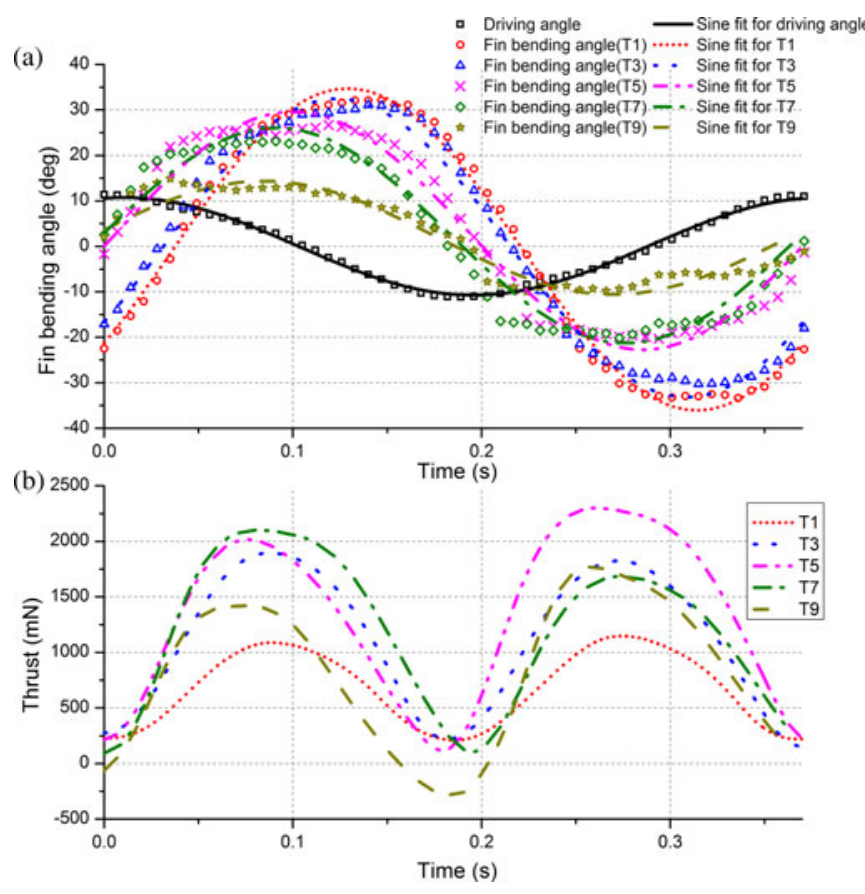

Fig. 7. Fin-bending angle, driving angle, and thrust results in the case of F3, at $2.7 \mathrm{~Hz}$. (a) Driving angle and fin-bending angle. (b) Measured thrust.

The relationship between the driving and fin-bending angles is presented in Fig. 7(a). The driving angle behaved like a pure sinusoid. Similarly, the fin-bending angle also behaved like a sinusoid, but with a plateau around the peak of the sinusoid. This phenomenon occurred when the driving angle was close to zero. As the stiffness of the caudal fin decreased, the duration of the plateau also increased, as shown in Fig. 7(a). Because the bending characteristics of caudal fins depend on their shapes, this plateau phenomenon can be described differently when considering different shapes of caudal fins. Even though the bending angle does not behave like an exact sinusoid, we can define the phase difference. The stiffness of the fin decreases from T9 to $\mathrm{T} 1$, as stated in Table I. As the stiffness of a caudal fin decreases, the phase difference increases. Fig 7(b) shows the thrust measurements for fins with varying stiffness. As stiffness increases, the peaks in the thrust increase. However, note that in the case of T9, as the stiffness of the fin increases, a negative thrust starts to occur at the bottom peak.

In the case of T5, we observe the largest average thrust and no-negative thrust in the cycle, as shown in Table IV. In this case, the phase difference is close to $\pi / 2$. This is defined as the half-pi phase delay condition. At the peak driving angle, the finbending angle is zero, which means that the fin is in line with the peduncle, as shown in Fig. 6(a). In other words, when the phase difference is $\pi / 2$ and the driving speed is maximized, the amplitude of the fin-bending angle is maximized. The largest peak fin-bending angle occurs in the case of $\mathrm{T} 1$, without this implying that it generates the largest average thrust. The largest peak in thrust occurs in the case of T5, while negative thrust occurs in the case of $\mathrm{T} 9$ at the bottom peak of the driving angle.
TABLE IV

Phase Difference $\xi$ AND Average Thrust at $2.7 \mathrm{~Hz}$ (F3 CASE)

\begin{tabular}{cccc}
\hline \hline Feature & Parameter & Phase difference $(\xi)$ & Thrust $(\mathrm{mN})$ \\
\hline \multirow{2}{*}{ Thrust min } & T1 & $0.67 \pi$ & 737.8 \\
& T3 & $0.60 \pi$ & 1009.1 \\
Thrust Max & T5 & $0.48 \pi$ & 1327.0 \\
& T7 & $0.46 \pi$ & 1188.7 \\
& T9 & $0.34 \pi$ & 802.0 \\
\hline \hline
\end{tabular}

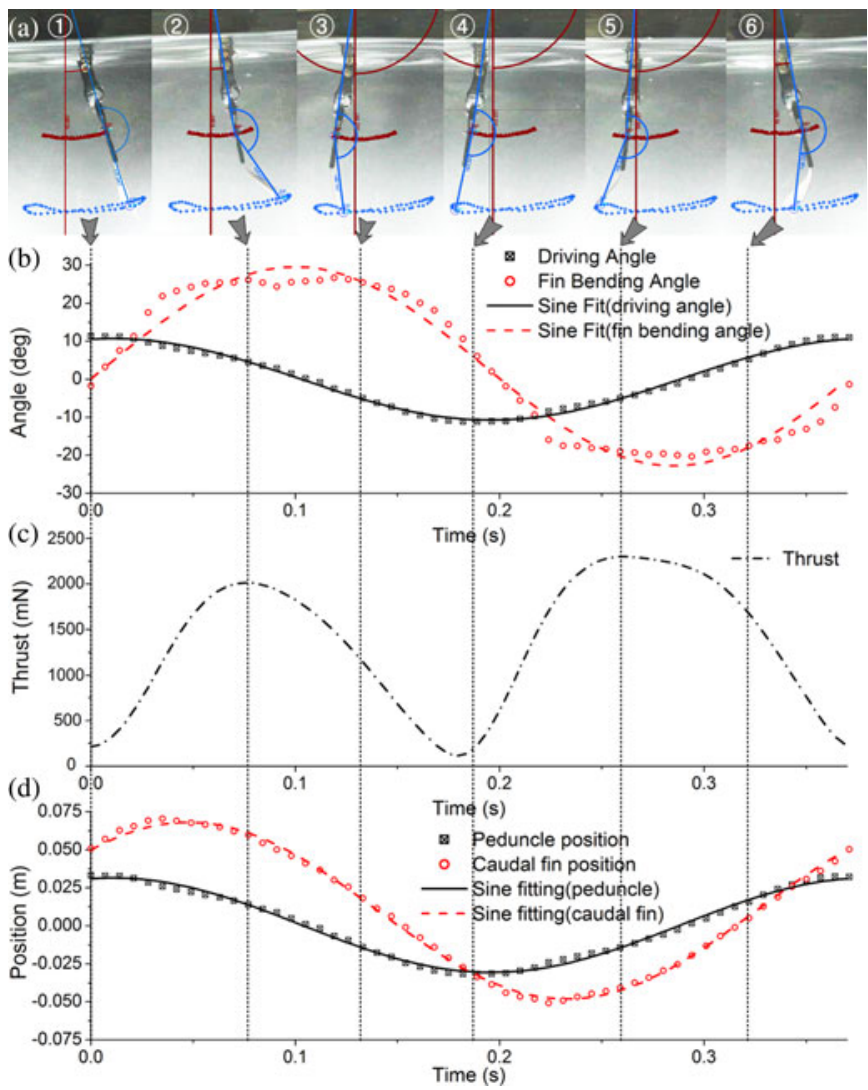

Fig. 8. Results for the caudal fin T5 with delta-shape II (F3), which corresponds to the maximum-thrust condition at $2.7 \mathrm{~Hz}$. (a) Caudal fin movements in the time frame. (b) Driving angle and fin-bending angle with the sine fitting graph (the phase difference between the two angle graphs is $87^{\circ}(\fallingdotseq 0.48 \pi)$ ). (c) Thrust results. (d) Horizontal ( $y$-axis) positional change (the phase difference between the two positions is $36^{\circ}(\fallingdotseq 0.20 \pi)$ ).

Specifically, in Fig. 8(b), we present the case of fin T5 with delta-shape II (F3), which is the fin that generated the maximum thrust for the driving frequency of $2.7 \mathrm{~Hz}$. The square dots denote the driving angle of the peduncle, and the circular dots denote the fin-bending angle. The driving angle oscillates between $+11^{\circ}$ and $-11^{\circ}$, while the fin-bending angle oscillates between $+26^{\circ}$ and $-26^{\circ}$. The two angles have been fitted to a sine curve. Note that the driving and fin-bending angles have a phase difference of $87^{\circ}(\fallingdotseq 0.48 \pi)$. When the driving angle (square dots) is close to zero, as shown in Fig. 8(a)-(2), the peduncle is close to its maximum velocity, and the fin-bending angle is near the maximum, because the phase difference is close to $90^{\circ}$. At this condition, the instant thrust is maximized. As the fin rotates 


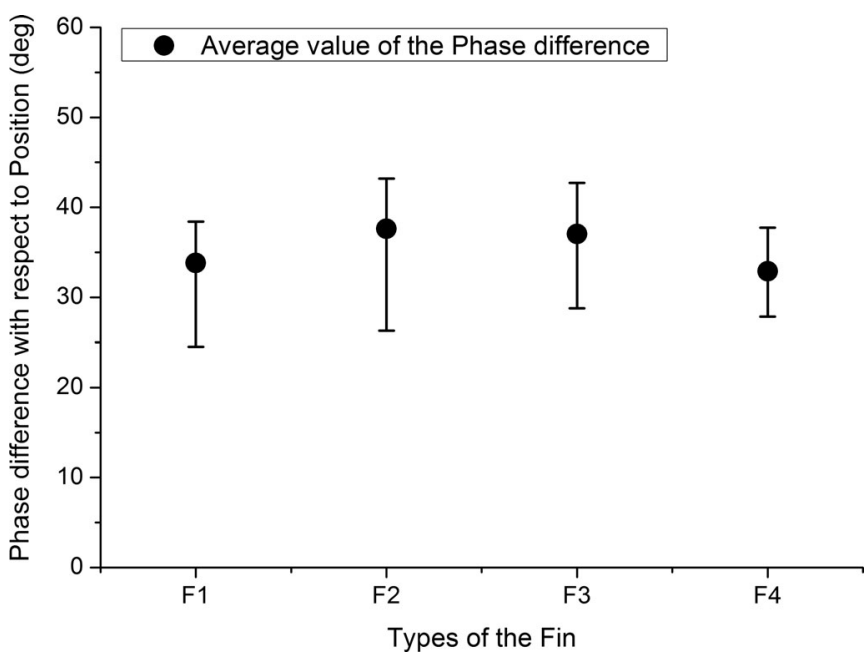

Fig. 9. Phase difference with respect to position, for each type of caudal fin, for cases that the maximum thrust is achieved, regardless of frequency.

clockwise, both the driving and fin-bending angles decrease, while maintaining the phase difference. Fig. 8(a)-(3) shows the case of a reduced bending angle and reduced thrust. When the fin reaches the minimum driving angle, as shown in Fig. 8(a)-(4), the velocity is close to zero, and the fin-bending angle is close to zero and changes from positive to negative. In this case, the instant thrust is at its minimum. For a compliant fin that generates the maximum thrust at a certain frequency, this is a typical relationship between the driving angle, fin-bending angle, and thrust. Note that if the phase difference changes, the bending angle will no longer be at its maximum when the velocity is at its maximum. In addition, where the velocity is close to zero, the bending angle direction will not change when the driving angle is at its peak. This could adversely affect thrust generation.

When describing the oscillatory motion of fins, positions are more widely used than angles. Fig 8(d) shows the $y$-positions of the endpoint of the peduncle (square dots), and the endpoint of the caudal fin (circular dots). The endpoints exhibit sinusoidal motion and the phase difference between the positions of the peduncle and the caudal fin in the $y$-axis was $36^{\circ}(\fallingdotseq 0.20 \pi)$. This phenomenon is also observed in nature. In general, the positions of the peduncle and fluke of a cetacean exhibit a phase difference of $34^{\circ}$, which for a driving frequency, regardless of the speed, is the average value of the observed data (ranging from $18.9^{\circ} \pm 5.9^{\circ}$ to $48.7^{\circ} \pm 26.9^{\circ}$ ) [31]. In Fig. 9, we plot the phase difference with respect to position, for all conditions that generate the maximum thrust for a certain compliant fin, regardless of the frequency. The ranges of the phase difference were from $24.5^{\circ}$ to $43.2^{\circ}$. This proves that our results are similar to those obtained through biological observation. Note that a phase difference of $34^{\circ}(\fallingdotseq 0.20 \pi)$ in the position is equivalent to a phase difference of $90^{\circ}(\fallingdotseq 0.5 \pi)$ in the angle.

To verify the half-pi phase delay condition, we present the results of the experiments described in Section III. In Fig. 10, we plot the normalized thrust values (maximum, minimum, and intermediate) with respect to phase difference, obtained by changing the shape and stiffness of the fins at various frequencies. The
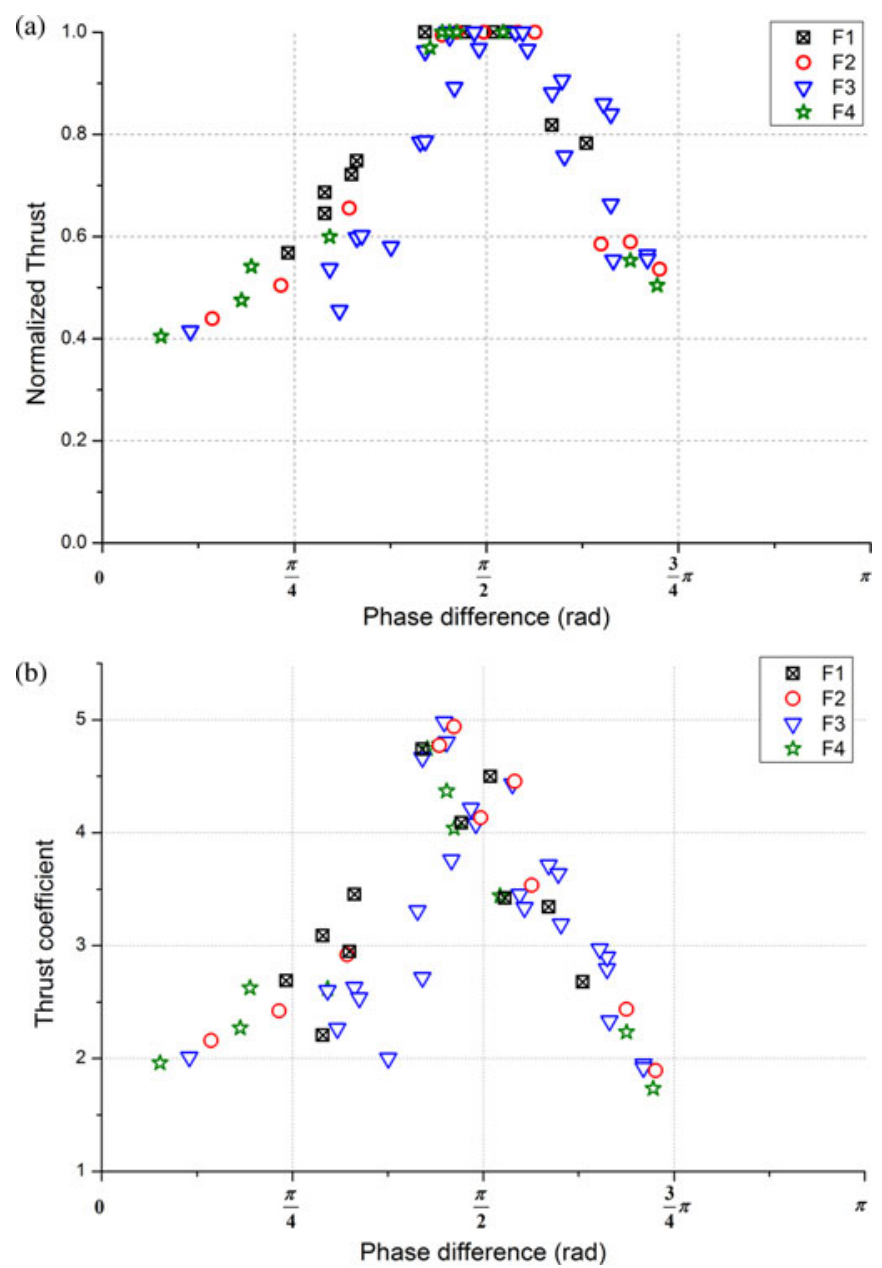

Fig. 10. (a) Normalized thrust values for all frequencies and all types of caudal fins, with respect to phase differences. The normalized thrust is maximized at $\pi / 2\left(=90^{\circ}\right)$. (b) Thrust coefficient for all frequencies and all types of caudal fins, with respect to phase differences. The symbols “ $₫$, , “०," “ $\nabla$, , and “沱” represent the normalized thrust corresponding to F1, F2, F3, and F4, respectively.

thrust values were normalized using the approach described in Section III-A. Even though the shape of the caudal fin changes, which changes the bending characteristics, the half-pi phase delay condition remains valid. Fig. 10(a) shows the normalized thrust values for all frequencies and all types of caudal fins with respect to phase differences. Based on this plot, the maximum normalized thrust is obtained at $\pi / 2\left(=90^{\circ}\right)$. This result can be verified in terms of thrust coefficient. The thrust coefficient is calculated based on the experimental results

$$
C_{T}=\frac{T}{0.5 \rho U^{2} A}
$$

where $\rho$ is the density of water, $U$ is the velocity of the caudal fin, $A$ is the platform area of the caudal fin, and $T$ is the thrust. Fig. 10(b) shows the thrust coefficient with respect to phase differences for all frequencies and all types of caudal fins.

Fig. 11(a) shows the increase of the phase difference as the frequency increases for the case of F3T5. The phase difference reaches $\pi / 2$ at the frequency of $2.75 \mathrm{~Hz}$. To verify that this is indeed the maximum-thrust condition, we measured the thrust 


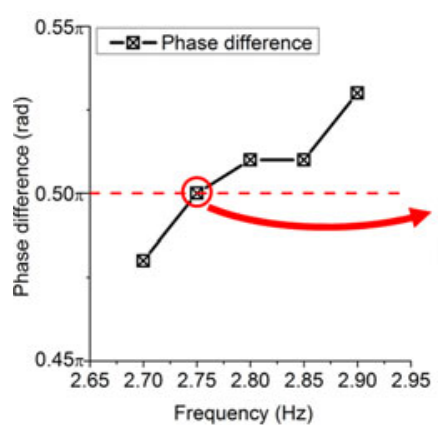

(a)

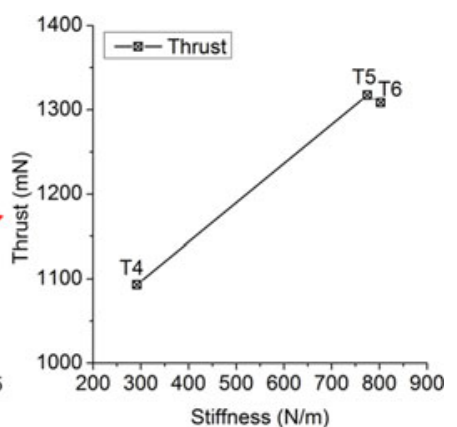

(b)
Fig. 11. (a) Phase difference in the case of F3T5 according to the frequency. (b) Thrust in the case of T4, T5, and T6 at $2.75 \mathrm{~Hz}$, when the phase difference of F3T5 is $\pi / 2\left(=90^{\circ}\right)$.
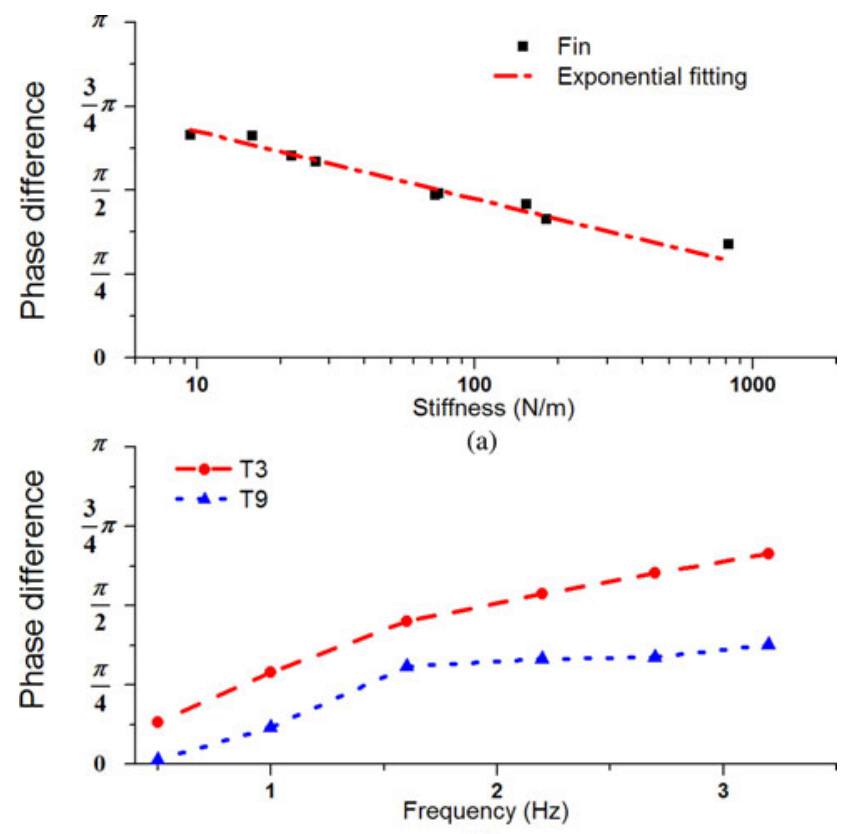

(b)

Fig. 12. (a) Phase difference versus stiffness at $2.7 \mathrm{~Hz}$, and relationship with the exponential fitting line. (b) Phase difference versus frequency.

generated by the fin at $2.75 \mathrm{~Hz}$ for T4 and T6 cases as well. As shown in Fig 11(b), the thrust of the T5 case is higher than the T4 case, which is more compliant than T5 and the T6 case, which is stiffer than T5.

Fig. 12(a) shows how the phase difference changes with varying stiffness, when the frequency is $2.7 \mathrm{~Hz}$. Fig. 12(b) shows for certain compliant fins (T3, T9), how the phase difference changes with varying frequency. The phase difference decreases as stiffness increases; the decrease is proportional to an exponential function of the stiffness of the caudal fin

$$
\xi=C e^{-D k}
$$

where $\xi$ is the phase difference, $k$ is the stiffness, and $C$ and $D$ are constants. For low values of stiffness, the phase difference is sensitive to changes in the stiffness. Conversely, for high values of stiffness, the phase difference changes little when varying the stiffness. On the other hand, the phase difference increases linearly with the oscillating frequency.

\section{Simple Model of Compliant Fin}

In general, the thrust of actual and robotic fish is estimated using either a hydrodynamic model or a numerical method. However, for robotic fish with a compliant fin, the thrust information is embedded in the bending of the fin. If we model the fin with a simple beam model, then we can derive the required force to bend the beam, and estimate the thrust generated by the fin. We will use this estimated thrust value to determine the compliance of a fin, which leads to a maximum thrust for a certain driving frequency.

In the experiments, we used four types of caudal fins: rectangular, triangular, and two delta-shaped fins. To simplify the model, we assumed that the caudal fin was a rectangular beam with average flexural stiffness $E I$. The average flexural stiffness $E I$ of the caudal fin can be calculated by integrating $E I(x)$

$$
E I(x)=\frac{1}{x_{\max }} \int_{0}^{x_{\max }} E I(x) d x
$$

where $E$ is the Young's modulus of the beam, and $I(x)$ is the area moment of inertia of the different shaped beam along the length of the caudal fin (chordwise direction). We ignore the bending of the fin in other directions (spanwise direction), and assume it bends only along the longitudinal direction.

As shown in Fig. 13(a), the bending motion of a caudal fin is similar to a flexible beam bending with a large deflection. The pseudo-rigid-body method (PRBM) is a simple method that is used to analyze a large deflection of a beam, under a point force applied at one end of the beam [37]. In PRBM, the fin is modeled as a rigid link connected to a peduncle with a pin joint and torsional spring, as shown in Fig. 13(b). Using this model, we estimate the deflection path and force-deflection relationships of a flexible beam. The actual hydrodynamic force is assumed a point force at the end of the beam, even though it is distributed along the beam. Moreover, the direction of the force $F$ is assumed perpendicular to the line $A B$.

The pseudo-rigid-body model is applied to the compliant fin in a quasi-static manner, and the motion of the caudal fin is analyzed frame by frame. The instant thrust is obtained by projecting the bending force at each frame to the $x$-axis.

In Fig. 13(c), $\Theta$ is the pseudo-rigid-body angle

$$
\Theta=\tan ^{-1} \frac{b}{a-l(1-\gamma)}
$$

where $\gamma$ is a characteristic radius factor, $a / l=1-\gamma(1-\cos \Theta)$, and $b / l=\gamma \sin \Theta$. These parameters can be determined by numerical methods. $F$ is the load that is applied to the pseudorigid-body (compliant fin)

$$
F=\frac{E I K_{\Theta} \Theta}{l^{2} \cos (\Theta-\varphi)} .
$$




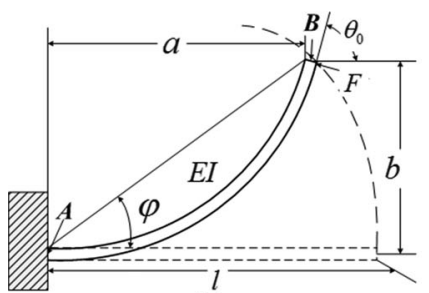

(a)

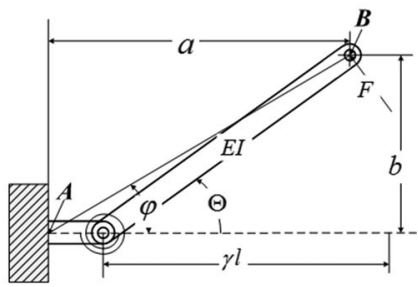

(b)

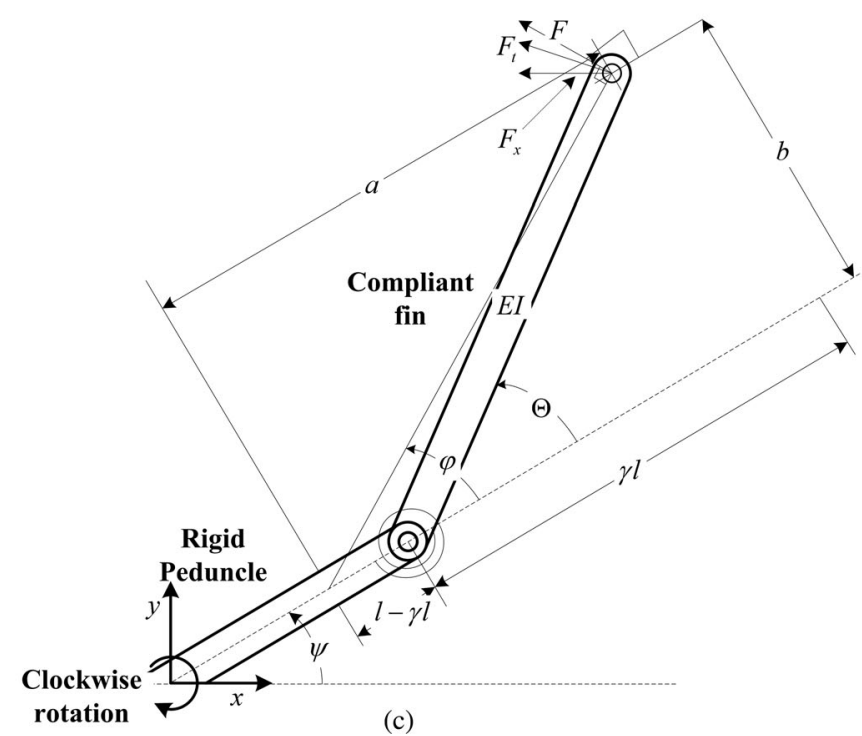

Fig. 13. (a) Flexible caudal fin model and (b) pseudo-rigid-body model of a caudal fin (redrawn from Howell [37]). (c) Pseudo-rigid-body model with the driving part being a rigid peduncle.

$F_{t}$ is the transverse component of the load according to the pseudo-rigid link

$$
F_{t}=\frac{E I K_{\Theta} \Theta}{l^{2}}=F \cos (\Theta-\varphi)
$$

where $E$ is the Young's modulus of the beam, $I$ is the area moment of inertia of the beam, $K_{\Theta}$ is the nondimensionalized torsional spring constant, and $l$ is the length of the beam. When the maximum pseudo-rigid-body angle does not exceed approximately $60^{\circ}, K_{\Theta}$ is 2.676 , and $\gamma$ is 0.852 [37].

The pseudo-rigid body angle $\Theta$ can be obtained from the bending angle $\varphi$, which is computed from the kinematical condition

$$
\Theta=\varphi+\sin ^{-1}\left(\frac{1-\gamma}{\gamma} \cos \varphi\right) .
$$

Thrust can be determined from the bending force $F$, which is in the negative $x$-direction, as shown in Fig. 13(c). After the bending force is obtained, the thrust can be computed by projecting it to the $x$-axis

$$
F_{x}=F \sin (\psi+\varphi) .
$$

The thrust is calculated by projecting the bending force on to the $x$-axis. However, there are cases where the thrust cannot be estimated with the beam-bending model. When the peduncle changes direction, the compliant fin also changes its direction
TABLE V

MEAsured Thrust, Estimated THRUST, AND PHASE DifFERENCE $\xi$ FOR THE MAXIMUM-THRUST CASE

\begin{tabular}{cccccccc}
\hline & & & & & \\
\hline
\end{tabular}

Measured: measured thrust (mN), Estimated: estimated thrust $(\mathrm{mN})$

after a certain period, meaning that for a certain period, the peduncle and fin move in opposite directions. In this case, the amount of bending of the fin does not represent the amount of thrust generated by the fin. This phenomenon occurs when the phase difference is not close to $\pi / 2$. For this reason, we estimate the thrust when the phase difference is equal to $\pi / 2$, which is the maximum-thrust case at each frequency.

To validate this model, at each frequency, we compared the estimated maximum thrust with the measured maximum thrust. The estimated maximum thrust was computed using the proposed model for a given Young's modulus, thickness, and length of the caudal fin. The fin-bending and driving angles were extracted from the video, and were used during the computation. Table V represents the experimental results for the maximumthrust case at each frequency. As seen in Table V, when the frequency is below $1.5 \mathrm{~Hz}$, the thrust is maximized when $\mathrm{T} 1$ is used. However, because the phase difference of $\mathrm{T} 1$ at 0.5 and $1.0 \mathrm{~Hz}$ is not close to $\pi / 2$, the estimated average thrust at these frequencies is not valid. Therefore, the estimations at 0.5 and $1.0 \mathrm{~Hz}$ were excluded. The measured and estimated data were compared with respect to frequency as shown in Fig. 14 for the case of F3. We selected the data from the compliant fin that had the maximum thrust for a given driving frequency. Overall, the estimated results were in good agreement with the measured results.

\section{Application to the Design of a Compliant Fin}

To design a compliant fin that maximizes thrust, we need to calculate the thrust generated by the designed compliant fin under a specific condition. However, as stated in Section I, the 


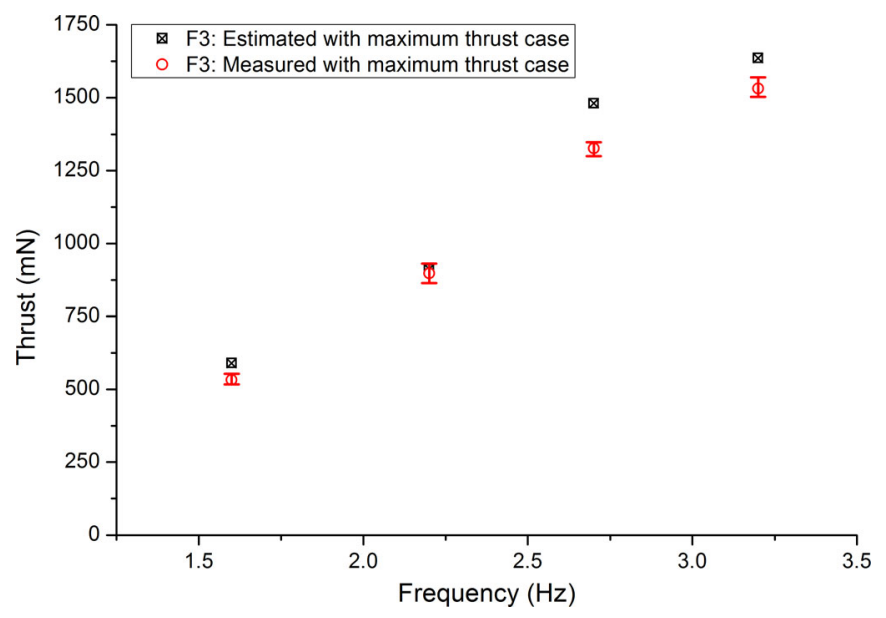

Fig. 14. Comparison between measured and estimated results, with respect to frequency, in the case of maximum thrust (F3).

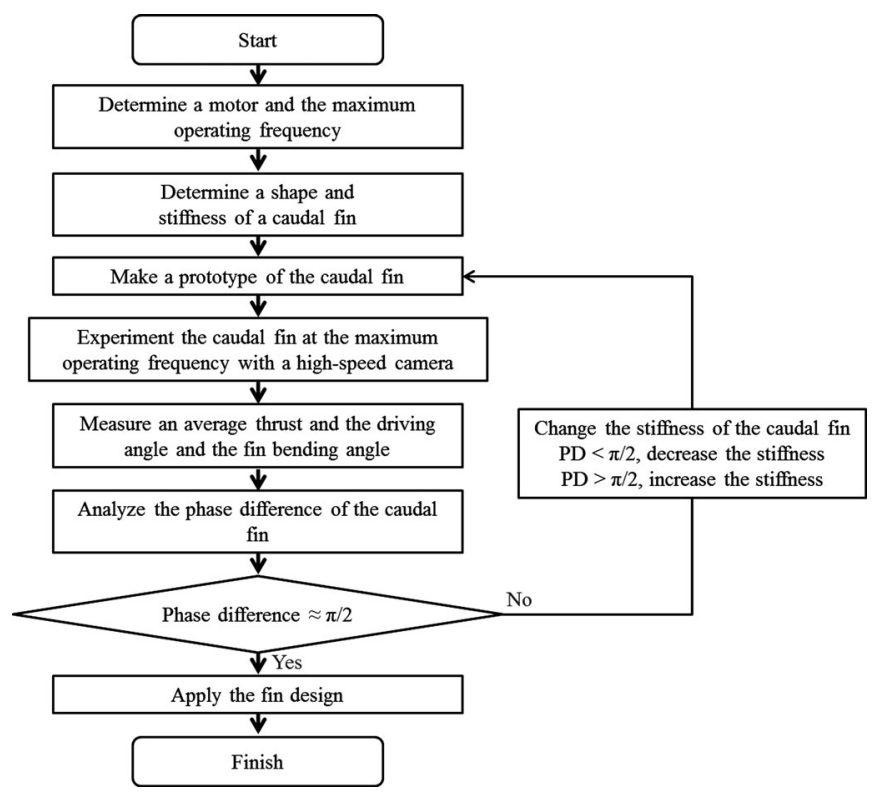

Fig. 15. Application process of the half-pi phase delay condition to design a caudal fin under the zero velocity condition and the fast-start condition.

thrust generated by the compliant fin cannot be calculated without experimental measurements of the kinematics. Therefore, the design of a compliant fin requires applying a trial and error procedure involving a large amount of experiments. On the other hand, the half-pi phase delay condition is a simple design guideline that determines whether the designed fin maximizes thrust. The value of the phase difference tells us whether the compliant fin needs to be stiffer or softer to increase the thrust. Specifically, if the phase difference is less than $\pi / 2$, the fin needs to be softer. Conversely, if the phase difference is larger than $\pi / 2$, the fin needs to be stiffer. Therefore, this kinematic condition can be used as a guideline to drastically reduce the number of experiments, which in turn enables the experimental design of a compliant fin.

The process of designing the compliant fin based on the halfpi phase delay condition under the zero velocity condition and the fast-start condition is presented as a flowchart in Fig. 15 . Details on how to apply the result to design a compliant caudal fin are as follows. First, we determine the parameters oscillating frequency and amplitude, which are related to the operating condition and motor specifications, respectively. The maximum operating frequency is an important factor, because it represents the kinematic condition that maximizes the thrust of a propulsion system with a compliant fin, while the zero velocity condition is satisfied.

After choosing the motor, the next step is to determine the shape and material of a caudal fin. In general, the shape of the robotic caudal fin approximates the actual shape of a caudal fin. Previous research showed that a delta-shaped fin is better than a rectangular or a triangular fin [21]. The desired compliance of the caudal fin is affected by the operating frequency. When the operating frequency is high, the stiffness of the fin also needs to be high.

The next step is to create a prototype of a caudal fin, and conduct experiments to measure the fin-bending and driving angles. Through this analysis, the phase difference of the proposed caudal fin is obtained. According to our results, to maximize thrust, the phase difference should be $\pi / 2$. If the phase difference is smaller than $\pi / 2$, the stiffness of the caudal fin needs to decrease. If the phase difference is larger than $\pi / 2$, the stiffness of the caudal fin needs to increase. Based on the analysis, a new prototype of the caudal fin is produced, and the experimental procedure is repeated until the optimal stiffness of the caudal fin that maximizes thrust is obtained. If the phase difference is close to $\pi / 2$, it implies that the optimal condition that maximizes the thrust of a propulsion system with a compliant fin, for a certain frequency, while scarifying the zero velocity condition, is obtained.

\section{CONCLUSION}

In this paper, we presented a simple method to identify the condition for maximizing the thrust generated by a compliant fin propulsion system, for a certain undulation frequency, while satisfying the zero velocity condition. Various studies have shown that the compliance of an undulating fin improves the performance of the fin, but the optimal condition has not yet been found. We investigated the thrust generated by a propulsion system with a single oscillating motor and a compliant caudal fin. We prepared four types of caudal fins (rectangular, triangular, and two delta-shaped) with various stiffness values and different ARs, and measured the thrust while driving the fins at different frequencies. We simplified the motion of the caudal fin by assuming that the driving and fin-bending angles follow a sinusoidal pattern, that the fin bends with a single curvature, and that the slope between the peduncle and fin is equal to zero. Under these simplifications, the phase difference between the driving and fin-bending angles was defined. The phase differences measured through kinematic analysis were compared with the measured thrust values. The results show that when maximum thrust is achieved, the fin-bending angle lags behind the driving angle by $\pi / 2$. This half-pi phase delay condition is simple and has been observed in biology [31], [32]. 
This condition implies that the fin bends to its maximum, when the peduncle is at its maximum speed. When the peduncle slows down as the driving angle reaches its peak, the fin straightens and changes its direction of bending as soon as the peduncle changes its direction of motion. In our experiments, we used different compliant fins at a driving frequency of $2.7 \mathrm{~Hz}$, and observed that the maximum thrust generated by the fin that satisfied the half-pi phase delay condition was twice as large as the thrust generated by most compliant fins, and 60\% larger than the thrust generated by the stiffest fin.

The maximum thrust of a robotic fish with a compliant fin can be estimated by calculating the force required to bend the fin. Under the half-pi phase delay condition, the projection of the bending force on the centerline of the body of the robot is the thrust generated by the fin. A flexible beam model and the PRBM were used to calculate the bending force that deflects the caudal fin. Although this method requires prior knowledge of the kinematics of the fin, and can only be used to estimate the maximum thrust, it does not require use of any fluid-related properties, e.g., drag and lift.

Because the current state of the art in compliant fin analysis requires experimental data to calculate thrust, finding the optimal condition of a fin directly is not possible. Using the half-pi phase delay condition, we can determine whether the compliance of a fin will generate the maximum thrust for a certain driving frequency, when satisfying the zero velocity condition. It is yet unknown if the half-pi phase delay condition will hold at a speed. Lauder showed that when the propulsive surface is quite flexible, the kinematics and pattern of forces change a lot if the foil is moving at its self-propelled speed or not. However, since the fins used in our experiments are at least 100 times stiffer than that of Lauder's experiments, it is likely that the flow condition has little effect on our results. However, when the robotic fish swim at a speed, stiffer caudal fin would be needed to maintain the half-pi phase delay condition. Further research is needed to verify whether this condition would hold for nonzero velocity condition and for more flexible fins that are closer to that of a real fish.

For a compliant fin to be optimal for multiple operating frequencies, the stiffness of the fin should be actively controllable. Further research on controllable stiffness fin could enable a robotic fish that adapts the stiffness of the fin according to the operating frequency. This phase difference criterion is nondimensional, which means that it can be used in a wide range of cases as a simple design guideline. This guideline is an important step toward establishing a design method to optimize the compliance of a robotic fish fin.

\section{ACKNOWLEDGMENT}

The authors would like to thank Prof. G. V. Lauder and Prof. H. Choi for their encouragement and constructive comments.

\section{REFERENCES}

[1] J. Liang, T. Wang, and L. Wen, "Development of a two-joint robotic fish for real-world exploration," J. Field Robot., vol. 28, no. 1, pp. 70-79, 2011.
[2] D. S. Barrett, "Propulsive efficiency of a flexible hull underwater vehicle,' Ph.D. dissertation, Dept. Ocean Eng., Mass. Inst. Technol., Cambridge, MA, 1996.

[3] H. Morikawa, S. Nakao, S.-I. Kobayashi, and H. Wada, "Experimental study on oscillating wing for propulsor with bending mechanism modeled on caudal muscle-skeletal structure of tuna," JSME Int. J., Series C: Mech. Syst., Mach. Elem. Manuf., vol. 44, no. 4, pp. 1117-1124, 2001.

[4] R. Fan, J. Yu, L. Wang, G. Xie, Y. Fang, and Y. Hu, "Optimized design and implementation of biomimetic robotic dolphin," in Proc. IEEE Int. Conf. Robot. Biomimetics, 2005, pp. 484-489.

[5] K. H. Low, "Modelling and parametric study of modular undulating fin rays for fish robots," Mech. Mach. Theory, vol. 44, no. 3, pp. 615-632, Mar. 2009.

[6] C. J. Esposito, J. L. Tangorra, B. E. Flammang, and G. V. Lauder, "A robotic fish caudal fin: Effects of stiffness and motor program on locomotor performance," J. Exp. Biol., vol. 215, no. 1, pp. 56-67, Jan. 2012.

[7] J. Tangorra, C. Phelan, C. Esposito, and G. Lauder, "Use of biorobotic models of highly deformable fins for studying the mechanics and control of fin forces in fishes," Integr. Comput. Biol., vol. 51, no. 1, pp. 176-189, Jul. 2011.

[8] G. V. Lauder, J. Lim, R. Shelton, C. Witt, E. Anderson, and J. L. Tangorra, "Robotic models for studying undulatory locomotion in fishes," Marine Technol. Soc. J., vol. 45, no. 4, pp. 41-55, 2011.

[9] P. V. y Alvarado and K. Youcef-Toumi, "Design of machines with compliant bodies for biomimetic locomotion in liquid environments," J. Dyn. Syst., Meas., Control, vol. 128, no. 1, pp. 3-13, Mar. 2006.

[10] P. Valdivia, Y. Alvarado, and K. Youcef-Toumi, "Performance of machines with flexible bodies designed for biomimetic locomotion in liquid environments," in Proc. IEEE Int. Conf. Robot. Autom., Apr. 2005, pp. 3324-3329.

[11] H. E. Daou, T. Salumae, A. Ristolainen, G. Toming, M. Listak, and M. Kruusmaa, "A bio-mimetic design of a fish-like robot with compliant tail," presented at the Int. Workshop Bio-Inspired Robots, Nantes, France, 2011.

[12] W. S. Chu, K. T. Lee, S. H. Song, M. W. Han, J. Y. Lee, H. S. Kim, M. S. Kim, Y. J. Park, K. J. Cho, and S. H. Ahn, "Review of biomimetic underwater robots using smart actuators," Int. J. Precis. Eng. Manuf., vol. 13, no. 7, pp. 1281-1292, 2012.

[13] K. H. Low and C. W. Chong, "Parametric study of the swimming performance of a fish robot propelled by a flexible caudal fin," Bioinsp. Biomimetics, vol. 5, no. 4, p. 046002, 2010. Available: http://iopscience.iop.org/1748-3190/5/4/046002.

[14] I. Yamamoto, Y. Terada, T. Nagamatu, and Y. Imaizumi, "Propulsion system with flexible/rigid oscillating fin," IEEE J. Oceanic Eng., vol. 20, no. 1, pp. 23-30, Jan. 1995.

[15] G. Lauder and P. Madden, "Learning from fish: Kinematics and experimental hydrodynamics for roboticists," Int. J. Autom. Comput., vol. 3, no. 4, pp. 325-335, Oct. 2006.

[16] G. V. Lauder, E. J. Anderson, J. Tangorra, and P. G. A. Madden, "Fish biorobotics: Kinematics and hydrodynamics of self-propulsion," J. Exp. Biol., vol. 210, no. 16, pp. 2767-2780, Aug. 2007.

[17] M. Vanella, T. Fitzgerald, S. Preidikman, E. Balaras, and B. Balachandran, "Influence of flexibility on the aerodynamic performance of a hovering wing," J. Exp. Biol., vol. 212, no. 1, pp. 95-105, Jan. 2009.

[18] F. E. Fish, M. K. Nusbaum, J. T. Beneski, and D. R. Ketten, "Passive cambering and flexible propulsors: Cetacean flukes," Bioinsp. Biomimetics, vol. 1, no. 4, pp. S42-S48, 2006.

[19] B. Ahlborn, D. G. Harper, R. W. Blake, D. Ahlborn, and M. Cam, "Fish without footprints," J. Theor. Biol., vol. 148, no. 4, pp. 521-533, 21. 1991.

[20] B. Ahlborn, S. Chapman, R. Stafford, and R. Harper, "Experimental simulation of the thrust phases of fast-start swimming of fish," J. Exp. Biol., vol. 200, no. 17, pp. 2301-2312, Sep. 1997.

[21] D. Kim and M. Gharib, "Characteristics of vortex formation and thrust performance in drag-based paddling propulsion," J. Exp. Biol., vol. 214, no. 13, pp. 2283-2291, Jul. 2011.

[22] M. Nakabayashi, R. Kobayashi, S. Kobayashi, and H. Morikawa, "Bioinspired propulsion mechanism using a fin with a dynamic variableeffective-length spring: Evaluation of thrust characteristics and flow around a fin in a uniform flow," J. Biomech. Sci. Eng., vol. 4, no. 1, pp. 82-93, 2009.

[23] T. M. Huh, Y. J. Park, and K. J. Cho, "Design and analysis of a stiffness adjustable structure using an endoskeleton," Int. J. Precis. Eng. Manuf., vol. 13, no. 7, pp. 1255-1258, 2012.

[24] T. Y.-T. Wu, "Swimming of a Waving Plate," J. Fluid Mech., vol. 10, no. 3, pp. 321-344, 1961.

[25] M. J. Lighthill, "Aquatic animal propulsion of high hydromechanical efficiency," J. Fluid Mech., vol. 44, no. 2, pp. 265-301, 1970. 
[26] Z. Chen, S. Shatara, and X. Tan, "Modeling of biomimetic robotic fish propelled by an ionic polymer-metal composite caudal fin," IEEE/ASME Trans. Mechatronics, vol. 15, no. 3, pp. 448-459, Jun. 2010.

[27] F. Liu, K.-M. Lee, and C.-J. Yang, "Hydrodynamics of an undulating fin for a wave-like locomotion system design," IEEE/ASME Trans. Mechatronics, vol. 17, no. 3, pp. 554-562, Jun. 2012.

[28] G. Seet, K. H. Low, C. W. Chong, and C. Zhou, "An improved semiempirical model for a body and/or caudal fin (BCF) fish robot," in Proc. IEEE Int. Conf. Rob. Autom., May 2010, pp. 78-83.

[29] M. Bergmann and A. Iollo, "Modeling and simulation of fish-like swimming," J. Comput. Phys., vol. 230, no. 2, pp. 329-348, Jan. 2011.

[30] I. Borazjani and F. Sotiropoulos, "On the role of form and kinematics on the hydrodynamics of self-propelled body/caudal fin swimming," J. Exp. Biol., vol. 213, no. 1, pp. 89-107, 2010.

[31] F. E. Fish, J. E. Peacock, and J. J. Rohr, "Stabilization mechanism in swimming odontocete cetaceans by phased movements," Marine Mammal Sci., vol. 19, no. 3, pp. 515-528, 2003.

[32] S. E. Spagnolie, L. Moret, M. J. Shelley, and J. Zhang, "Surprising behaviors in flapping locomotion with passive pitching," Phys. Fluids, vol. 22, no. 4, pp. 041903-1-041903-20, 2010.

[33] M. Sfakiotakis, D. M. Lane, and J. B. C. Davies, "Review of fish swimming modes for aquatic locomotion," IEEE J. Oceanic Eng., vol. 24, no. 2, pp. 237-252, Apr. 1999.

[34] J. Yu, Y. Hu, J. Huo, and L. Wang, "Dolphin-like propulsive mechanism based on an adjustable Scotch yoke," Mech. Mach. Theory, vol. 44, no. 3, pp. 603-614, Mar. 2009.

[35] F. L. Vanderplank, "Air-speed/wing-tip speed ratios of insect flight," Nature, vol. 165 , no. 4203, pp. 806-807, 1950.

[36] E. D. Tytell, "Do trout swim better than eels? Challenges for estimating performance based on the wake of self-propelled bodies," Exp. Fluids, vol. 43, no. 5, pp. 701-712, 2007.

[37] L. L. Howell, Compliant Mechanisms. New York: Wiley, 2001.

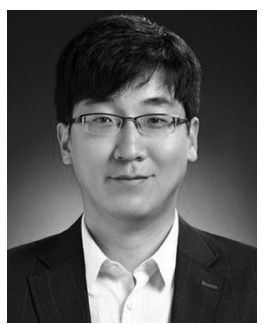

Yong-Jai Park (S'10) received the B.S. degree in mechanical and aerospace engineering from Seoul National University, Seoul, Korea, in 2004, where he is currently working toward the Ph.D. degree with the BioRobotics Laboratory, Department of Mechanical and Aerospace Engineering.

He has worked for a semiconductor company in a managerial position. His research interests include robotic fish, biologically inspired robotics, surgical robots, and novel mechanisms.

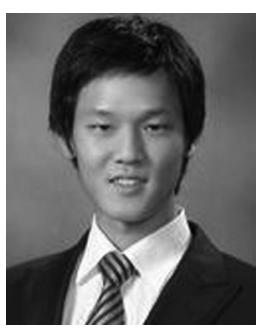

Useok Jeong (S'10) received the B.S. degree in mechanical and aerospace engineering from Seoul National University, Seoul, Korea, in 2010, where he is currently working toward the Ph.D. degree with the BioRobotics Laboratory, Department of Mechanical and Aerospace Engineering.

His research interests include robotic fish, biomimetic robots, and surgical robots with particular emphasis on human-robot interaction and actuators.

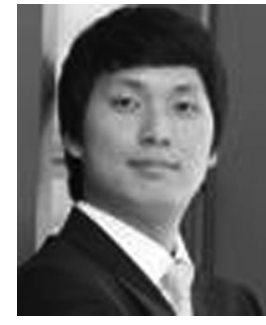

Jeongsu Lee received the Ph.D. degree in mechanical and aerospace engineering from Seoul National University, Seoul, Korea, in 2009, where he is currently working toward the M.S. degree with the Micro Fluid Mechanics Laboratory, Department of Mechanical and Aerospace Engineering.

His research interests center around biofluid dynamics.

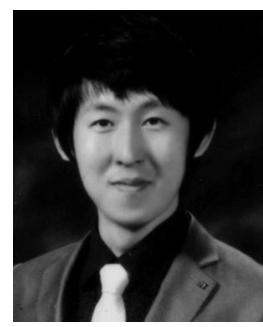

Seok-Ryung Kwon received the B.S. degree in mechatronics from the Korea University of Technology and Education, Cheonan, Korea, in 2010. He is currently working toward the M.S. degree with the BioRobotics Laboratory, Department of the Mechanical and Aerospace Engineering, Seoul National University, Seoul, Korea.

His research interests include robotic fish, robot manipulators, surgical robots, and humanoid robots with particular emphasis on bioinspired human friendly robots.

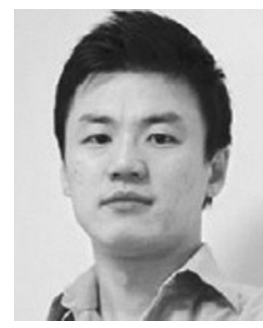

Ho-Young Kim received the B.S degree from Seoul National University, Seoul, Korea, in 1994 and the M.S. and Ph.D. degrees from the Massachusetts Institute of Technology, Cambridge, in 1996 and 1999, respectively.

He is currently an Associate Professor of mechanical and aerospace engineering and the Director of the Micro Fluid Mechanics Laboratory, Seoul National University.

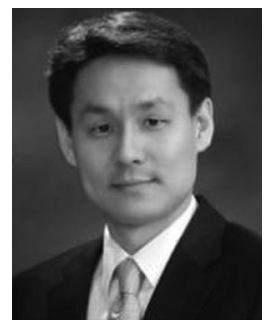

Kyu-Jin Cho (M'08) received the B.S and M.S. degrees from Seoul National University, Seoul, Korea, in 1998 and 2000, respectively, and the Ph.D. degree in mechanical engineering from the Massachusetts Institute of Technology, Cambridge, in 2007.

He was a Postdoctoral Fellow with Harvard Microrobotics Laboratory, Cambridge, until 2008. He is currently an Assistant Professor of mechanical and aerospace engineering and the Director of the BioRobotics Laboratory, Seoul National University. His research interests include biologically inspired robotics, robotic systems using smart actuators, novel mechanisms using smart structures, and rehabilitation and assistive robotics. 